${ }^{1}$ Departamento de Nutrición Diabetes y Metabolismo Escuela de Medicina, Pontificia Universidad Católica de Chile. Santiago de Chile.

${ }^{2}$ Universidad Diego Portales, Santiago, Chile

aMédico Cirujano, Magíster en Nutrición.

bBioquímico, PhD, MSc. 'Bioquímico, MSc.

Los autores declararon no tener conflictos de intereses, empleando el formulario "ICMJE Form for Disclosure of Potential Conflicts of Interest"

Recibido el 14 de marzo de 2013 aceptado el 13 de noviembre de 2013.

Correspondencia a:

Dr. Alberto Maiz.

Departamento de Nutrición, Diabetes y Metabolismo. Facultad de Medicina, Pontificia Universidad Católica de Chile. Marcoleta 367. Santiago, Chile. Teléfono: 562-23543862 maiz@med.puc.cl

\section{Xantomatosis cerebrotendinosa: aspectos fisiopatológicos, clínicos y genéticos}

\author{
YUDITH PREISS ${ }^{1,2, a}$, JOSÉ L. SANTOS ${ }^{2, b}$, \\ SUSAN V. SMALLEY ${ }^{2, \mathrm{c}}$, ALBERTO MAIZ ${ }^{2}$
}

\section{Cerebrotendinous xanthomatosis: physiopathology, clinical manifestations and genetics}

Cerebrotendinous xanthomatosis $(C T X)$ is a rare autosomal recessive disease, caused by genetic deficiency of the 27-hydroxylase enzyme (encoded by CYP27A1). It plays a key role in cholesterol metabolism, especially in bile acid synthesis and in the 25-hydroxylation of vitamin D3 in the liver. Its deficiency causes reduced bile acid synthesis and tissue accumulation of cholestanol. Clinical manifestations are related to the presence of cholestanol deposits and include tendon xanthomas, premature cataracts, chronic diarrhea, progressive neurologic impairment and less frequently coronary heart disease, early onset osteoporosis and abnormalities in the optic disk and retina. An early diagnosis and treatment with quenodeoxycholic acid may prevent further complications, mainly neurological manifestations. This review summarizes cholesterol metabolism related to bile acid synthesis, physiopathology, biochemistry and treatment of cerebrotendinous xanthomatosis.

(Rev Med Chile 2014; 142: 616-622)

Key words: Cholestanol; Chenodeoxycholic acid; Cholesterol; CYP27A1; Xanthomatosis, cerebrotendinous.
L a xantomatosis cerebrotendinosa (XCT) es una rara enfermedad autosómica recesiva, causada por mutaciones en el gen CYP27A1, que codifica para la enzima mitocondrial 27esterol hidroxilasa. Esta enzima participa en el metabolismo del colesterol y otros esteroles, especialmente en el proceso de síntesis de ácidos biliares. Cataliza el paso inicial en la oxidación de la cadena lateral de esteroles intermediarios en la formación de ácidos biliares. Además, participa junto a otras hidroxilasas en la 25-hidroxilación de la vitamina $\mathrm{D} 3^{1}$. Las mutaciones con efecto funcional en CYP27A1 determinan una disminución en la cantidad de enzima o una actividad defectuosa de la misma, provocando la disminución de la síntesis de ácidos biliares y el aumento del colestanol en el plasma y en tejidos, uno de los $5 \alpha$ dihidro derivados intermediarios en el metabolismo del colesterol $^{2-4}$.

Las manifestaciones clínicas se presentan en relación al depósito de colestanol en diferentes tejidos, como tendones, cristalino y sistema nervioso central ${ }^{4}$. Los pacientes inicialmente presentan diarrea durante la infancia y cataratas juveniles $y$, en etapas más avanzadas de la enfermedad, aparecen xantomas tendinosos, piramidalismo, signos de compromiso cerebeloso, convulsiones $y$, menos frecuentemente, cardiopatía coronaria $\mathrm{y}$ ateroesclerosis precoz $^{5,6}$. Otras manifestaciones menos frecuentes son alteraciones oftalmológicas del disco óptico y senescencia prematura de la retina $^{7-9}$. Además, en asociación a la deficiencia de 
25-hidroxi-vitamina $\mathrm{D}$, se ha descrito la existencia de osteoporosis precoz ${ }^{1}$.

En Chile existen dos publicaciones al respecto. La primera, del año 2009 sobre un caso con diagnóstico clínico y de laboratorio de $\mathrm{XCT}^{10}$. La segunda, del año 2011, informó sobre un paciente con xantomatosis severa, en el cual por medio del estudio genético se demostró la condición de heterocigoto compuesto para dos mutaciones diferentes en CYP27A1 causantes de XCT, así como otra alteración genética asociada a una hipercolesterolemia familiar con una mutación en el gen del receptor de LDL en estado heterozigoto ${ }^{11}$.

El presente trabajo tiene como objetivo realizar una revisión sobre la XCT, abarcando aspectos del metabolismo del colesterol en relación a la síntesis de ácidos biliares, conocimientos actuales sobre las mutaciones descritas y antecedentes sobre el desarrollo de la enfermedad y su tratamiento.

\section{Metabolismo del colesterol y ácidos biliares}

El colesterol participa en numerosos y esenciales procesos fisiológicos, tales como la biosíntesis de distintos productos con actividad biológica específica como son los ácidos biliares y las hormonas esteroidales. Por esta razón, es de gran importancia asegurar su disponibilidad a los tejidos ${ }^{12}$.

El colesterol hepático proviene de la síntesis endógena y en menor grado de la absorción intestinal y del transporte reverso mediado por las lipoproteínas de alta densidad (HDL) ya sea directamente (vía receptor SRB1) o indirectamente (vía receptores de LDL/IDL). El colesterol es eliminado por las heces a través de su conversión a ácidos biliares en el hígado. Además de ser un mecanismo de excreción del colesterol, los ácidos biliares promueven la absorción de lípidos dietarios, incluyendo vitaminas liposolubles y son ligandos de receptores nucleares que regulan la expresión de importantes genes involucrados en la homeostasis del colesterol. Existen dos principales vías en la síntesis de ácidos biliares: la "clásica" o "neutra" y la "alternativa" o "acídica". A la primera se la ha conocido como vía neutra dado que los precursores de ácidos biliares proceden de esteroides neutros, siendo la vía más importante cuantitativamente en la síntesis de ácidos biliares primarios. La vía alternativa se le llama acídica dado que sus precursores proceden de cascadas de intermediarios de ácidos carboxílicos ${ }^{13,14}$.
La vía clásica comienza con la $7 \alpha$-hidroxilación del colesterol por la enzima $7 \alpha$-hidroxilasa, siendo éste el paso limitante de la vía neutra. En cambio, en la vía acídica, el primer paso es la 27-hidroxilación del colesterol. La enzima 27-esterol hidroxilasa es la primera enzima de la vía acídica y se encuentra asociada a la membrana mitocondrial interna, expresándose en diferentes tejidos además del hígado. En este órgano tiene como función la degradación de la cadena lateral del colesterol y, a nivel extrahepático, de la degradación de colesterol celular para procesos metabólicos y estructurales ${ }^{13,14}$.

La 27-esterol hidroxilasa es una enzima con una secuencia de 33 aminoácidos de señalización a mitocondria, seguidos por una proteína madura de 498 aminoácidos. En ésta, se han identificado dos dominios funcionales: el sitio de unión a adrenodoxina y el sitio de unión al ligando heme ${ }^{15,16}$. La enzima es expresada en múltiples tejidos: hígado, pulmón, sistema nervioso central, duodeno, macrófagos, sangre y piel, entre otros ${ }^{3}$. En el hígado, donde ejerce su principal función, cataliza el primer paso en la oxidación de la cadena lateral del colesterol convirtiéndolo a productos 27-oxigenados, hidroxilando el grupo metilo de C-27, para formar un grupo carboxilo ${ }^{13,14}$. Además, participa en la 25-hidroxilación a nivel mitocondrial de la vitamina $\mathrm{D} 3^{12,17,18}$. La función extrahepática de la enzima estaría relacionada con la regulación y homeostasis del colesterol al actuar el 27 hidroxicolesterol como ligando de receptores nucleares. Este intermediario produce un feedback negativo, vía la Sterol Regulatory Element Binding Protein (SREBP), a la enzima hidroximetilglutaril co-enzima A reductasa (HMG-CoA reductasa), que es la enzima limitante en el proceso de biosíntesis del colesterol ${ }^{13}$.

\section{Fisiopatología de la xantomatosis cerebrotendinosa (XCT)}

El principal defecto metabólico en la XCT es la disminución de la síntesis de ácido quenodeoxicólico, causada por la ausencia o la disminución de la función de la enzima 27-esterol hidroxilasa. La disminución de la función de esta enzima a nivel hepático, dada por variaciones cuali o cuantitativas, determina una disminución en la síntesis de ácidos biliares, principalmente del ácido 
quenodeoxicólico y un aumento de la producción de intermediarios como el colestanol y alcoholes biliares. Esta disminución del pool de ácidos biliares, sería la razón por la cual la biosíntesis del colesterol se encuentra aumentada, debido al mecanismo de retroalimentación compensatorio, mediado por la enzima limitante $7 \alpha$-hidroxilasa, que se encuentra sobreestimulada. Existiría entonces en la XCT, una sobreproducción de colesterol y colestanol, sobretodo a partir de productos $7 \alpha$ hidroxilados (Figura 1).

Existen varios posibles mecanismos por los cuales la CYP27A1 podría participar en la eliminación de colesterol de las células extrahepáticas. De hecho, esta enzima es considerada de gran importancia en la homeostasis del colesterol en macrófagos y células endoteliales ${ }^{19-21}$. El 27-hidroxicolesterol es una molécula más polar que el colesterol, siendo eliminada de la célula de manera más eficiente. Se ha descrito que existe un flujo permanente de 27-hidroxicolesterol hacia el hígado, para convertirlo posteriormente en ácidos biliares. La ausencia de este mecanismo podría jugar algún rol en la ateroesclerosis prematura descrita en estos pacientes. Además, el 27 hidroxicolesterol es ligando de receptores nucleares de genes que codifican para proteínas de transporte de colesterol tipo $\mathrm{ABC}$, que están presentes en macrófagos, estimulando así el transporte reverso de colesterol vía HDL ${ }^{14,22}$.

En relación al depósito de colestanol en el sis- tema nervioso central (SNC), Salen et al postulan que es posible que la presencia de apolipoproteína $B$ detectada en el líquido cefalorraquídeo explique el paso anormal de lipoproteínas LDL, que pueden contener colestanol además de colesterol $^{23}$. Sin embargo, el año 2007 Bhattacharyya et al evaluaron en pacientes con diagnóstico de XCT, la absorción del colestanol dietario y el turnover del colesterol y colestanol tras la administración intravenosa de C14-colesterol. Se vio que no existe paso de colestanol exógeno hacia el cerebro ni hacia nervios periféricos ${ }^{24}$. La tesis más reciente es la de Björkhem et al, quienes postulan que el 7ahydroxy-4-cholesten-3-one, sería el principal precursor del colestanol, debido a la sobreactivación de la enzima 7 alfa hidroxilasa. Este metabolito, en condiciones normales, pasa la barrera hematoencefálica $^{25}$. Demostraron in vitro que células gliales y astrocitos podrían convertirlo en colestanol ${ }^{26,27}$. Aún falta evidencia in vivo que explique el depósito de estos metabolitos en el sistema nervioso central.

Como se mencionó anteriormente, CYP27A1 también tiene un rol en el metabolismo de la 25- $(\mathrm{OH})$-vitamina D3. La vitamina D3 o colecalciferol, es sintetizada en la piel y no presenta actividad biológica. Su metabolismo continúa en el hígado, tanto a nivel microsomal como mitocondrial. Se ha postulado que la osteoporosis precoz y más severa que se ve en algunos pacientes con XCT podría estar relacionada con la menor absorción de vitamina $\mathrm{D}$ debido a la alteración en

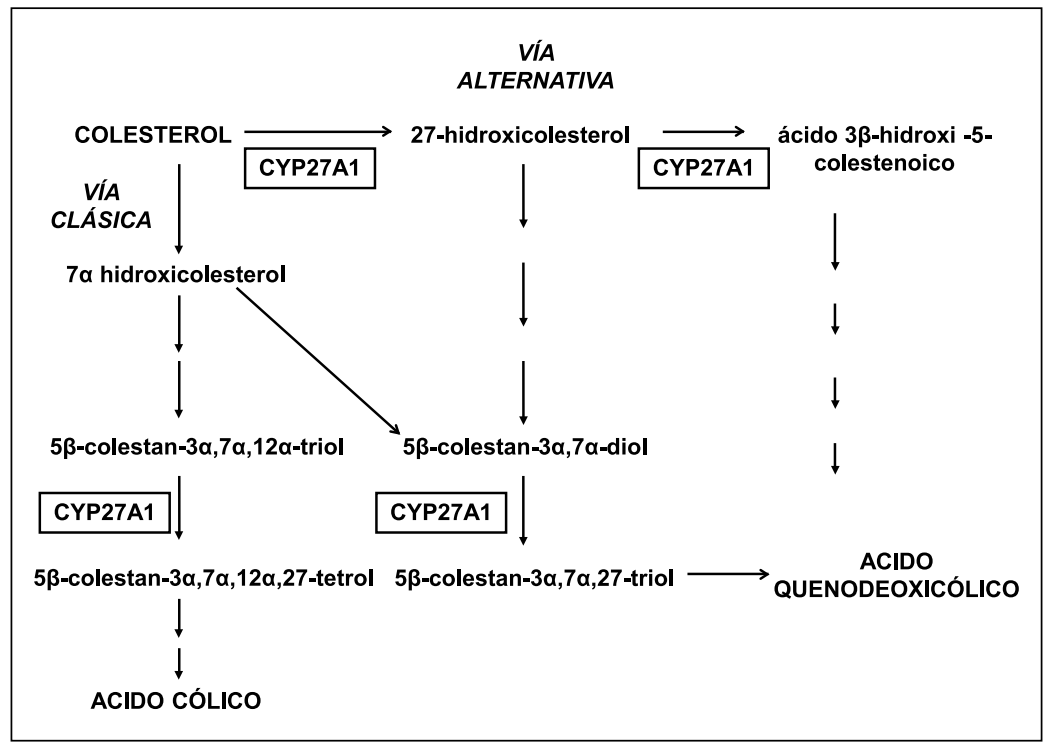

Figura 1. Principales vías involucradas en síntesis de ácidos biliares. Adaptado de Norlin M, Wikvall $\mathrm{K}^{14}$. 
la síntesis de ácidos biliares ${ }^{1}$. Sin embargo, el hecho de que la vitamina D3 sea sintetizada en la piel contradice aquella hipótesis. Ha sido comprobado que la afinidad enzimática para la hidroxilación de vitamina D3 es mayor para otras enzimas ${ }^{17,18}$ siendo esta diferencia la posible explicación del por qué no todos los pacientes con XCT presentan osteoporosis.

\section{Mutaciones en CYP27A1 causantes de XCT}

Hasta este momento, se han descrito aproximadamente unas 60 mutaciones para este gen ${ }^{28}$ y no se han descrito mutaciones en otros genes responsables de la $\mathrm{XCT}^{3}$. Es interesante destacar que no se ha especificado una relación clara entre el genotipo y el fenotipo en pacientes con XCT, existiendo una importante variabilidad intrafamiliar en las manifestaciones clínicas de la enfermedad ${ }^{5,15,28}$. CYP27A1 codifica para la enzima 27-esterol hidroxilasa, que pertenece a la subfamilia A, familia 27, del citocromo P-450. Se han descrito diferentes transcritos de este gen en humanos con diferente patrón de corte-empalme (splicing) (http://www.ensembl.org), ubicándose en la porción distal del brazo largo del cromosoma 2. El gen contiene 9 exones y 8 intrones y abarca $18,6 \mathrm{~Kb}$ de ADN. En la revisión de Gallus et $\mathrm{al}^{2}$ se afirma que la mayoría de las mutaciones generan una sustitución de un aminoácido, siendo menos frecuentes las mutaciones que generan directamente un codón de término prematuro o las que ocurren en sitios de splicing. Las mutaciones que afectan el splicing, pueden generar ya sea una eliminación de un exón o un cambio en el marco de lectura que podría llevar a un péptido enzimáticamente inactivo o a un codón de término prematuro, lo que a su vez podría desencadenar una rápida degradación del mRNA producido. En el caso de tratarse de mutaciones en la secuencia codificante del gen, éstas pueden afectar a su actividad principalmente por su efecto sobre los sitios de activación de la proteína ${ }^{1,2,15}$.

\section{Características clínicas de la XCT}

La enfermedad se presenta en la mayoría de los casos desde la infancia precoz. Diarrea, cataratas juveniles, signología neuro-psiquiátrica, principalmente de compromiso cerebelar y piramidal y xantomas tendinosos, son las principales manifestaciones (Tabla 1) ${ }^{28-30}$. Al menos la mitad de los pacientes debuta con cuadros de diarrea, durante el período de lactante, que son de difícil manejo y de curso crónico. Se desconoce la patogenia de la diarrea, sin embargo, se cree que el gran volumen de alcoholes biliares intraluminales jugaría un rol, al favorecer la motilidad, el traspaso de electrolitos desde el epitelio y el desequilibrio de la flora intestinal ${ }^{30,31}$.

El segundo signo precoz es la aparición de cataratas juveniles. Dotti et al, examinaron 13 pacientes con diagnóstico clínico y genético de XCT. Once de los trece pacientes presentaron cataratas cuya edad de aparición fluctuó entre los 10 y 30 años ${ }^{9}$. La prevalencia de cataratas varía entre 92 y $96 \%$ según las diferentes revisiones ${ }^{28,29}$.

Los xantomas tendinosos tienden a aparecer durante la segunda a tercera década de la vida siendo los sitios más frecuentemente afectados el tendón de Aquiles (Figura 2), los extensores de codo y mano, el patelar y a nivel cervical. Estos xantomas contienen tejido conectivo y células espumosas en cuyo interior hay principalmente colestanol y colesterol ${ }^{3,32,33}$.

La signología neurológica es frecuente en esta enfermedad. Se describe trastorno del desarrollo psicomotor, demencia, trastornos psiquiátricos, signos de focalización, signos piramidales y extrapiramidales, parkinsonismo y neuropatía periférica. Estos serían secundarios al depósito de material lipídico principalmente en hemisferios cerebelares, globus pallidus y pedúnculos cerebelares. En las imágenes, además, es común observar atrofia cerebral ${ }^{2}$. Se piensa que la atrofia y el daño axonal difuso sería secundario a la neurotoxicidad de los depósitos ${ }^{34,35}$.

\section{Tabla 1. Frecuencia de signología clásica en series} clínicas de xantomatosis cerebrotendinosa

\begin{tabular}{|lll|}
\hline & $\begin{array}{l}\text { Pilo B et } \\
\text { al (Espa- } \\
\tilde{\text { ña) }} \\
\text { (n = 25) }\end{array}$ & $\begin{array}{l}\text { Verrips A } \\
\text { et al (Ho- } \\
\text { landa) } \\
\text { (n = 54) }\end{array}$ \\
\hline Xantomas tendíneos & $14(56 \%)$ & $27(50 \%)$ \\
Cataratas precoces & $23(92 \%)$ & $52(96 \%)$ \\
Diarrea & $11(44 \%)$ & $19(35 \%)$ \\
Compromiso neurológico & $23(92 \%)$ & $51(94 \%)$ \\
\hline
\end{tabular}




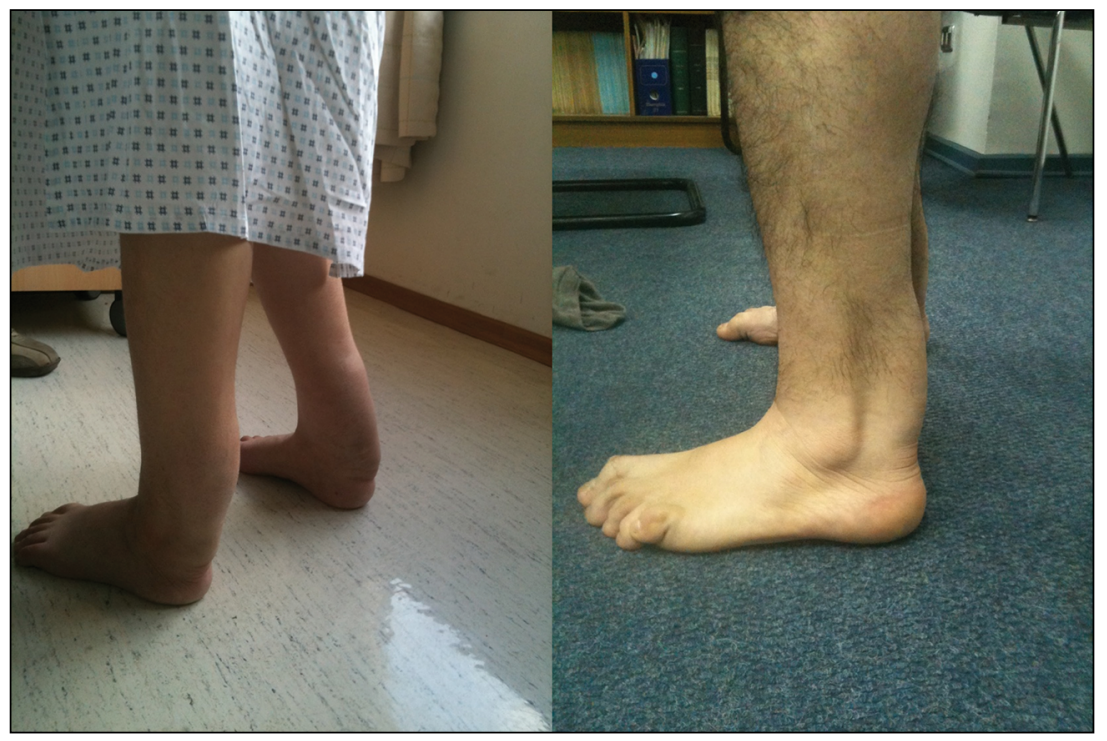

Figura 2. Xantomas tendíneos (tendón de Aquiles) en pacientes chilenos con Xantomatosis Cerebrotendinosa.
El diagnóstico de XCT se basa en la sospecha clínica ante la presencia de dos de los cuatro signos cardinales: diarreas sin causa aparente, xantomas tendinosos, cataratas precoces o signología neurológica ${ }^{28}$. El diagnóstico final se realiza con la constatación de niveles elevados de colestanol plasmático, prueba que no se encuentra aún disponible en nuestro país. El apoyo imagenológico con resonancia nuclear magnética de encéfalo y médula espinal puede aportar información para apoyar la sospecha. Imágenes con hiperintensidades en cerebelo, región periventricular, globus pallidus y núcleos dentados de cerebelo, son altamente sugerentes de XCT, en asociación con las manifestaciones clínicas descritas ${ }^{2}$. El estudio genético no es necesario en el proceso diagnóstico, ni existe evidencia que muestre asociación entre genotipos y posibles fenotipos y la evolución de la enfermedad ${ }^{5,15,28}$. Sin embargo, en nuestro país, sería la única manera disponible de asegurar el diagnóstico. Entre nuestros objetivos se encuentra el implementar la tecnología necesaria para medir niveles de colestanol plasmático, con el fin de apoyar el diagnóstico y tratamiento precoz y evitar complicaciones posteriores.

\section{Tratamiento}

El tratamiento de la enfermedad es el uso de ácido quenodeóxicolico (AQDC), el cual suprime la síntesis de ácidos biliares al generar feedback negativo sobre la enzima $7 \alpha$-hidroxilasa. Se ha demostrado que su administración logra normalizar los niveles de colestanol plasmático ${ }^{3,4,36}$. En los últimos años se ha descrito que la adición de estatinas al AQDC produciría un efecto sinérgico, al disminuir el pool de colesterol disponible, mejorando la respuesta clínica ${ }^{37,38}$. Los efectos del tratamiento con AQDC en cuanto a los resultados clínicos son controvertidos. Ha sido ampliamente demostrado que su uso en pacientes diagnosticados precozmente, previo a la aparición de depósitos de colestanol en el sistema nervioso central, evita la progresión de la enfermedad y las complicaciones neurológicas, cognitivas y osteotendíneas, que en general se manifiestan más tardíamente ${ }^{39,40}$. Sin embargo, es menos contundente la evidencia sobre la efectividad del tratamiento una vez presente el daño neurológico. Si bien se normalizan los niveles de colestanol, la evolución es variable, existiendo publicaciones que muestran desde regresión de la signología neurológica y del tamaño de xantomas ${ }^{28,37,41}$, hasta otras series en las cuales los pacientes, a pesar de recibir el AQDC, se mantuvieron estables o continuaron deteriorándose ${ }^{28,39,42}$. Entre éstas, la serie de pacientes españoles analizada por Pilo de la Fuente et al, quienes observaron que los años de sobrevida estaban relacionados con la precocidad del diagnóstico ${ }^{28}$. 
Debido a que ésta es una enfermedad autosómica recesiva, se recomienda estudiar a los familiares, pues en etapas tempranas de la vida puede ser asintomática, siendo el ácido quenodeoxicólico un tratamiento efectivo para la prevención de los depósitos de colestanol ${ }^{2}$. Desafortunadamente, la disponibilidad del fármaco en Chile es limitada. El AQDC ha sido declarada una molécula "huérfana" por su escaso uso a nivel mundial y pocos laboratorios la siguen comercializando, lo que determina un alto costo para los pacientes. En Chile, no es importada por laboratorios ni por las grandes cadenas farmacéuticas. En el año 2010, dentro del contexto del estudio de pacientes chilenos con diagnóstico de XCT llevado a cabo por este grupo, el Instituto de Salud Pública aprobó la importación de la materia prima y su comercialización bajo recetario magistral preparado en cápsulas de $250 \mathrm{mg}$, con el fin de realizar seguimiento y de fiscalizar su adecuada formulación para el consumo de los pacientes. La dosis recomendada es de 750 $\mathrm{mg}$ al día y $15 \mathrm{mg}$ por $\mathrm{kg}$ de peso en niños.

\section{Referencias}

1. Moghadasian MH. Cerebrotendinous xanthomatosis: clinical course, genotypes and metabolic backgrounds. Clin Invest Med 2004; 27 (1): 42-50.

2. Gallus GN, Dotti MT, Federico A. Clinical and molecular diagnosis of cerebrotendinous xanthomatosis with a review of the mutations in the CYP27A1 gene. Neurol Sci 2006; 27 (2): 143-9.

3. Björkhem I, Hansson M. Cerebrotendinous xanthomatosis: an inborn error in bile acid synthesis with defined mutations but still a challenge. Biochem Biophys Res Commun 2010; 396 (1): 46-9.

4. Björkhem I, Boberg KM. Inborn errors in bile and biosynthesis and storage of sterols other than cholesterol. En: Scriver CR, Beaudet AL, Sly WS, Valle D, Editores, The Metabolic and Molecular Basis of Inherited Disease, 7th ed. McGraw- Hill, New York; 1995. p 207399.

5. Dotti MT, Garuti R, Calandra S, Federico A. Clinical and genetic variability of CTX. Eur J Neurol 1996; 3 [Suppl 5]: 12 .

6. Dotti MT, Mondillo S, Plewnia K, Agricola E, Federico A. Cerebrotendinous xanthomatosis: evidence of lipomatous hypertrophy of the atrial septum. J Neurol 1998; 245: 723-26.

7. Matsumuro K, Takahashi K, Matsumoto H, Okatsu Y, Kuriyama M. A case of cerebrotendinous xanthomatosis with convulsive seizures. Rinsho Shinkeigaku 1990; 30 (2): 207-9.

8. Dotti MT, Federico A, Signorini E, Caputo N, Venturi C, Filosomi G, et al. Cerebrotendinous xanthomatosis (van Bogaert-Scherer- Epstein disease): CT and MR findings. AJNR Am J Neuroradiol 1994; 15: 1721-6.

9. Dotti MT, Rufa A, Federico A. Cerebrotendinous xanthomatosis: heterogeneity of clinical phenotype with evidence of previously undescribed ophthalmological findings. J. Inherit. Metab Dis 2001; 24: 696-706.

10. Filippi J, Irarrázaval S, Peredo P, Mellado P. Xantomatosis cerebrotendínea: Una causa de aumento de volumen bilateral del tendón de Aquiles. Caso clínico. Rev Med Chile 2009; 137: 815-20.

11. Huijgen R, Stork AD, Defesche JC, Peter J, Alonso R, Cuevas A, et al. Extreme xanthomatosis in patients with both familial hypercholesterolemia and cerebrotendinous xanthomatosis. Clin Genet 2012; 81 (1): 24-8.

12. Cortés VA, Busso D, Mardones P, Maiz A, Arteaga A, Nervi F, et al. Advances in the physiological and pathological implications of cholesterol. Biol Rev Camb Philos Soc 2013; 88: 825-43.

13. Norlin M, von Bahr S, Bjorkhem I, Wikvall K. On the substrate specificity of human CYP27A1: implications for bile acid and cholestanol formation. J Lipid Res 2003; 44 (8): 1515-22.

14. Norlin M, Wikvall K. Enzymes in the conversion of cholesterol into bile acids. Curr Mol Med 2007; 7 (2): 199-218.

15. Lee MH, Hazard S, Carpten JD, Yi S, Cohen J, Gerhardt GT, et al. Fine-mapping, mutation analyses, and structural mapping of cerebrotendinous xanthomatosis in U.S. pedigrees. J Lipid Res 2001; 42 (2): 159-69.

16. Björkhem I, Leitersdorf E. Sterol 27-hydroxylase deficiency: A rare cause of xanthomas in normocholesterolemic humans. Trends Endocrinol Metab 2000; 11 (5): 180-3.

17. Sawada N, Sakaki T, Ohta M, Inouye K. Metabolism of vitamin D (3) by human CYP27A1. Biochem Biophys Res Commun 2000; 273: 977-84.

18. Cheng JB, Levine MA, Bell NH, Mangelsdorf DJ, Russell DW. Genetic evidence that human CYP2R1 is a key vitamin D 25- hydroxylase. Proc Natl Acad Sci USA 2005; 101: 711-15.

19. Lund E, Andersson O, Zhang J, Babiker A, Ahlborg G, Diczfalusy $\mathrm{U}$, et al. Importance of a novel oxidative mechanism for elimination of intracellular cholesterol in humans. Arterioscler Thromb Vasc Biol 1996; 16: 208-12.

20. Babiker A, Andersson O, Lund E, Xiu RJ, Deeb S, Reshef $\mathrm{A}$, et al. Elimination of cholesterol in macrophages 
and endothelial cells by the sterol 27-hydroxylase: comparison with high density lipoprotein-mediated reverse cholesterol transport. J Biol Chem 1997; 272: 26253-61.

21. Björkhem I, Diczfalusy U, Lütjohann D. Removal of cholesterol from extrahepatic sources by oxidative mechanisms. Curr Opin Lipidol 1999; 10: 161-5.

22. Reiss AB, Martin KO, Javitt NB , Martin DW, Grossi EA, Galloway AC. Sterol 27-hydroxylase: high activity in vascular endothelium. J Lipid Res 1994; 35: 1026-30.

23. Salen G, Berginer V, Shore V, Horak I, Horak E, Tint GS, et al. Increased concentrations of cholestanol and apolipoprotein B in the cerebrospinal fluid of patients with cerebrotendinous xanthomatosis. N Engl J Med 1987; 316: 1233-8.

24. Bhattacharyya AK, Lin DS, Connor WE. Cholestanol metabolism in patients with cerebrotendinous xanthomatosis: absorption, turnover, and tissue deposition, J. Lipid Res 2007; 48: 185-92.

25. Björkhem I. Cerebrotendinous xanthomatosis, Curr Opin Lipidol 2013; 24: 283-7.

26. Meaney S, Heverin M, Panzenboeck U, Ekström L, Axelsson M, Andersson U, et al. Novel route for elimination of brain oxysterols across the blood-brain barrier: conversion into 7a-hydroxy-3-oxo-4-cholestanoic acid, J. Lipid Res 2007; 48: 944-51.

27. Panzenboeck U, Andersson U, Hansson M, Sattler W, Meaney S, Björkhem I. On the mechanism of cerebral accumulation of cholestanol in cerebrotendinous xanthomatosis. J Lipid Res 2007; 48: 1167-74.

28. Pilo-de-la-Fuente B, Jiménez-Escrig A, Lorenzo JR, Pardo J, Arias M, Ares-Luque A, et al. Cerebrotendinous xanthomatosis in Spain: clinical, prognostic, and genetic survey. Eur J Neurol 2011; 18 (10): 1203-11.

29. Verrips A, Hoefsloot LH, Steenbergen GC, Theelen JP, Wevers RA, Gabreëls FJ, et al. Clinical and molecular genetic characteristics of patients with cerebrotendinous xanthomatosis. Brain 2000; 123 (Pt5): 908-19.

30. Verrips A, van Engelen BG, Wevers RA, van Geel BM, Cruysberg JR, van den Heuvel LP, et al. Presence of diarrhea and absence of tendon xanthomas in patients with cerebrotendinous xanthomatosis. Arch Neurol 2000; 57 (4): 520-4.

31. van Heijst AF, Wevers RA, Tangerman A, Cruysberg JR, Renier WO, Tolboom JJ. Chronic diarrhea as a dominating symptom in two children with cerebrotendinous xanthomatosis. Acta Paediatr 1996; 85: 932-6.
32. Smithard A, Lamyman MJ, McCarthy CL, Gibbons CL, Cooke PJ, Athanasou N, et al. Cerebrotendinous xanthomatosis presenting with bilateral Achilles tendon xanthomata.Skeletal Radiol 2007; 36 (2): 171-5.

33. von Bahr S, Movin T, Papadogiannakis N, Pikuleva I, Rönnow P, Diczfalusy U, et al. Mechanism of accumulation of cholesterol and cholestanol in tendons and the role of sterol 27-hydroxylase (CYP27A1). Arterioscler Thromb Vasc Biol 2002; 22: 1129-35.

34. Soffer D, Benharroch D, Berginer V. The neuropathology of cerebrotendinous xanthomatosis revisited: a case report and review of the literature. Acta Neuropathol 1995; 90 (2): 213-20.

35. De Stefano N, Dotti MT, Mortilla M, Federico A. Magnetic resonance imaging and spectroscopic changes in brains of patients with cerebrotendinous xanthomatosis. Brain 2001; 124 (Pt 1): 121-31.

36. Pilo de la Fuente B, Sobrido MJ, Girós M, Pozo L, Lustres M, Barrero F, et al. Usefulness of cholestanol levels in the diagnosis and follow-up of patients with cerebrotendinous xanthomatosis. Neurologia 2011; 26 (7): 397-404.

37. Watts GF, Mitchell WD, Bending JJ, Reshef A, Leitersdorf E, et al. Cerebrotendinous xanthomatosis: a family study of sterol 27-hydroxylase mutations and pharmacotherapy. QJM 1996; 89 (1): 55-63.

38. Verrips A, Wevers RA, Van Engelen BG, Keyser A, Wolthers BG, Barkhof F, et al. Effect of simvastatin in addition to chenodeoxycholic acid in patients with cerebrotendinous xanthomatosis. Metabolism 1999; 48 (2): 233-8.

39. Berginer VM, Gross B, Morad K, Kfir N, Morkos S, Aaref S, et al. Chronic diarrhea and juvenile cataracts: think cerebrotendinous xanthomatosis and treat. Pediatrics 2009; 123 (1): 143-7.

40. Monson DM, DeBarber AE, Bock CJ, Anadiotis G, Merkens LS, Steiner RD, et al. Cerebrotendinous xanthomatosis: a treatable disease with juvenile cataracts as a presenting sign. Arch Ophthalmol 2011; 129 (8): 1087-8.

41. Berginer VM, Salen G, Shefer S. Long-term treatment of cerebrotendinous xanthomatosis with chenodeoxycholic acid. N Engl J Med 1984; 311 (26): 1649-52.

42. Burnett JR, Moses EA, Croft KD. Brown AJ, Grainger K, Vasikaran SD, et al. Clinical and biochemical features, molecular diagnosis and long-term management of a case of cerebrotendinous xanthomatosis. Clin Chim Acta 2001; 306 (1-2): 63-9. 